A 3D virtual learning environment to foster communication for long term ill children

Peer-reviewed author version

DI FIORE, Fabian; JORISSEN, Pieter; VANSICHEM, Gert \& VAN REETH, Frank (2007) A 3D virtual learning environment to foster communication for long term ill children. In: TECHNOLOGIES FOR E-LEARNING AND DIGITAL

ENTERTAINMENT, PROCEEDINGS. p. 92-103.

DOI: $10.1007 / 978-3-540-73011-8 \_12$

Handle: http://hdl.handle.net/1942/7798 


\title{
A 3D Virtual Learning Environment to Foster Communication for Long Term Ill Children
}

\author{
Fabian Di Fiore $^{1}$, Pieter Jorissen $^{1}$, Gert Vansichem $^{2}$, and Frank Van Reeth $^{1}$ \\ Hasselt University \\ Expertise Centre for Digital Media - IBBT \\ transnationale Universiteit Limburg \\ Wetenschapspark, 2 \\ BE-3590 Diepenbeek (Belgium) \\ http://www.edm. uhasselt.be \\ 2 ANDROME NV \\ Wetenschapspark, 4 \\ BE-3590 Diepenbeek (Belgium) \\ http://www . androme.com \\ fabian.difiore@uhasselt.be, pieter.jorissen@uhasselt.be, gvansichem@androme.com, \\ frank.vanreeth@uhasselt.be
}

\begin{abstract}
In this paper, we present a virtual learning environment which supports the learning process of long term sick children. By focusing on 3D Virtual Environments technology chronically ill students can still be 'telepresent' in their classroom.

Analysis of existing ICT-based solutions reveal weaknesses including the absence of social involvement, the high cost of developing, and mobility issues. Our system, however, is based on concrete user needs, is educational sound and relevant, and offers a scalable and affordable solution. To this end we incorporate innovative hardware, software and connectivity features, set in a user friendly user interface based on 3D technologies. We believe our system (re)establishes high quality communication between the sick children and their regular school and classroom learning environment. Furthermore, even after school pupils are attracted to meet each other in the virtual learning environment in order to communicate and share information.
\end{abstract}

Keywords: eHealth, eLearning, edutainment, Collaborative Learning, Virtual Interactive Communities

\section{Introduction}

Motivation. Health care, nowadays, is becoming less and less based in hospitals and increasingly home-based. Regular schools, however, are hardly able to set up instruction of high quality with these home-based children. 'Consultants' that support the pupils at home instead of the teacher do not present an optimal solution due to the lack of interaction with the teacher, and the irregular nature of the relationship between school and the home. Moreover, friendships between the ill child and the fellow pupils become more and more disintegrated.

It is our aim to re-establish especially the communication link between the sick pupils and the regular classroom in view of supporting high quality instructional scenarios. 


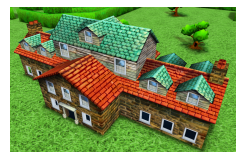

(a)

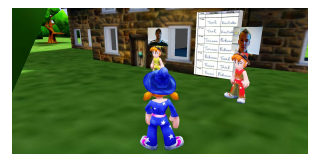

(b)

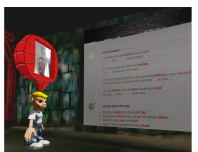

(c)

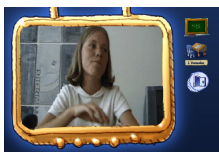

(d)

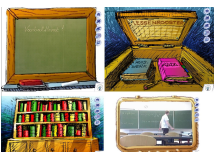

(e)

Fig. 1. Snapshots of our virtual learning environment. a) Virtual school building. b) Video-based avatars. c) Published assignment. d) Teacher's view in the classroom. e) Pupil's views of the classroom.

Contribution. We present here a technical solution that helps to establish high quality involvement of the long term sick children in a communication based scenario between the place where the child stays/has been moved and the original classroom/school setting. The communication provisions build on audio/video links (3D, very high to very low quality video links, synchronous versus asynchronous solutions), and will help to support educational scenarios (e.g. a one-to-one scenario) that foster particular learning processes (e.g., a school friend tutors the sick child in the context of a reading comprehension exercise; or a sick child develops answers for a work sheet presented in a classroom via a video image of the task form). Based on a preliminary analysis, the target age group for the project is school children between 8 and 12 years, and the actors supporting the child in its educational/medical context.

It is to be stressed that we do not focus on developing an electronic learning environment (ELE) such as Blackboard, WebCT, Anywize, etc. or a content management system (CMS). We rather focus on a solution that is able to be linked to existing ELE and CMS solutions. The system will also not focus on developing content. Users (e.g., teachers) are expected to input or refer to their available content, used during their normal classroom activities, in the educational scenarios.

Approach. Technically the main innovative challenge is to combine the best of 3D Virtual Environment technology (e.g., for representing the surrounding classroom scenery as well as navigation in the scenery) together with video-based rendering approaches (e.g., for representing the children as well as transmitting specific learning content) for realising a telepresence virtual learning environment. A series of examples are shown in Figure 1.

\section{Related Work}

During recent years, a variety of solutions, based on the integrated use of information and communication technologies (ICT), has been developed, implemented and tested in a variety of settings.

An advanced prototype solution has been developed in the USA in the PEBBLES project (Providing Education By Bringing Learning Environments to Stu- 
dents) 1. It comprises a social and technology solution that virtually places a child within the classroom by means of a robot which is present in the regular classroom, replacing the sick child. The robot can be controlled by the sick pupil from a distance. This solution enables to recreate an authentic setting since video, audio and documents can be transmitted from both sides. The robot is connected through a high-speed internet line to the tools in the hospital. The PEBBLES solution enables a strong synchronous and authentic 'presence' of the sick pupil in the original classroom setting. But the moment the sick pupil is no longer able to follow the lesson (lack of concentration, attack, treatment, ...) there is the problem of backing up the experiences being missed. Next, there is also the issue of mobility of the ICT-based solution. Another critical issue, hardly mentioned in the literature, is the high cost of developing and maintaining the PEBBLES-provision.

STARBRIGHT World (SBW) is a safe and secure online community where children living with serious illnesses can connect with each other. Children on SBW can chat, read and post to bulletin boards, email, search for friends with similar illnesses, participate in fun events and contests, surf pre-screen Web sites and play games [2. Analysis of the use of SBW, however, pointed out that the communicative possibilities seldom were used by the children: only $3 \%$ to $15 \%$ of the time was spent on communication 3 .

In the Flemish setting, a type of video phone has been promoted to support long term sick children to get in touch with their family and peers at school 4 . Other existing solutions in the Flemish setting build on extending desktop and laptop-based solutions with video phones and webcams. Although these solutions can support social presence, they offer basic video connection capabilities of rather low quality. In addition, each use of a video phone involves extra costs.

We have to mention that next to the use of these device, there is a promotion of the use of an electronic learning environment (ELE) through which the school, parents and pupils get more regularly in touch. However, these tools build heavily on text-based input and communication and are therefore less suited for younger students. Besides, they create a less authentic setting and hardly support the active and interactive involvement of the sick pupils.

\section{$3 \quad$ Virtual Learning Environment}

The virtual learning environment we envisage consists of a virtual community that can be shared by many users including the sick pupil, the teacher and the fellow pupils. Figure 2 gives an overview of our system. In the following subsections we discuss the underlying virtual interactive community framework, the functional aspects and the network architecture.

\subsection{Virtual Interactive Community}

Virtual Communities (VC) can be defined as a community of people sharing the same interests or ideas who are remotely present through the internet. The 


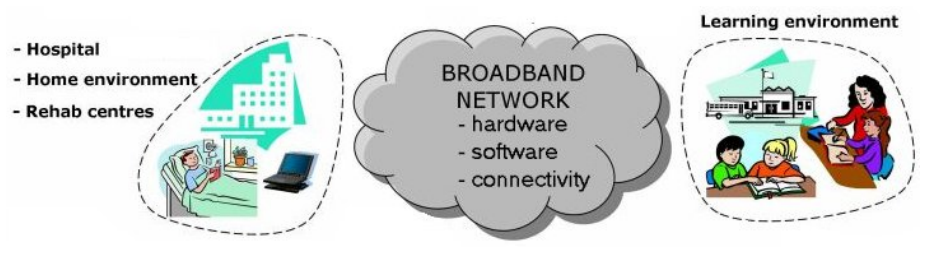

Fig. 2. Overview of our system.

virtual learning environment we envisage consists of a $3 \mathrm{D}$ virtual community in which chronically ill students can still be 'telepresent' in their classroom.

An initial user needs analysis [5] revealed that using 3D for e-learning applications is a flexible media solution that accelerates the knowledge transfer process and increases student self-sufficiency: (i) end-users prefer non-abstract environments, and they prefer seeing themselves in context in the space when interacting with others, and (ii) 3D visualisation of classrooms lends a more explorative, fun atmosphere to the learning environment. However, as interaction within this 3D learning environment should be intuitive and natural, the virtual environment should support communication (e.g., instant messaging, file transfer, audio/video communication), authentication (e.g., to determine identification, rights management, privacy), personalisation (e.g., virtual profile, visual representation), and presence (e.g., contact of buddy lists, online status).

In this work, we build further on the code framework developed by Quax et al. that allows for interacting in virtual environments [6].

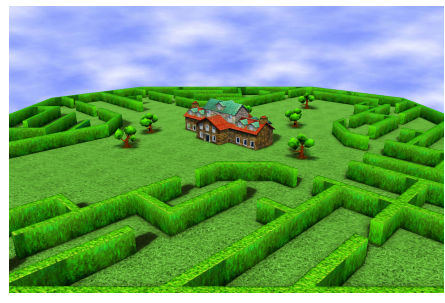

(a)

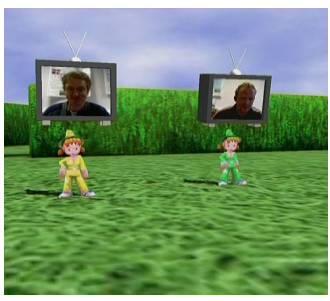

(b)

Fig. 3. Snapshots of the virtual learning environment. a) View of the entire world. b) The use of video stream texturing on animated avatars.

Virtual World. Upon starting the tool, the user automatically ends up in the $3 \mathrm{D}$ virtual environment. Taking into account that the community is childrenoriented, all its objects are created in cartoon style (see Figure 3).

Navigating in this virtual world happens by means of a graphical personification of the user, also called avatar. This corresponds to navigating in present-day 
games and on-line communities like 'Ice Age 2' and '(Teen) Second Life' and, therefore, fits in with their living environment. The environment also provides for community support functions like buddy lists (with classmates, teachers, parents), authentication \& authorisation functionalities providing the pupil with the same level of privacy, and rich presence (showing who is online, which mood, what kind of activities one is pursuing).

Furthermore, we integrated various synchronous communication components into the environment including audio conversation, video conferencing (with remotely controllable web cams), text chat and video-based avatars [6] (see also Figure $3(\mathrm{~b}))$.

The Pupil in the Virtual Classroom. By navigating through the virtual world and the school, pupils can enter the virtual classroom. Unlike the rest of the world, the classroom is represented by a fixed and static $3 \mathrm{D}$ view comprised of the most important parts of a real classroom (see Figure 4(a)).

Looking at the classroom we can distinguish a live video view of the class (top, left), a captured image of the blackboard (top, middle), the pupil's school desk (bottom), and the pupil's personal bookshelf (top, right). Furthermore, several function buttons can be noticed whose purpose is to (i) quickly launch specific tasks including scanning, transmitting or printing documents, capturing the blackboard, drawing attention, and (ii) jump to predefined views such as a close-up view of the blackboard, the personal bookshelf, opened school desk, live view of the classroom.

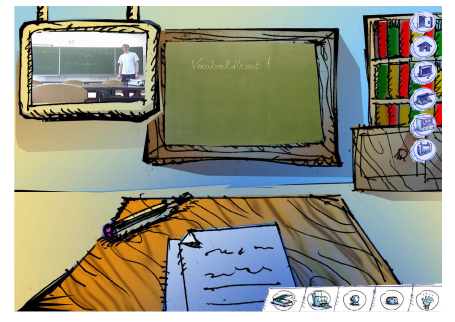

(a)

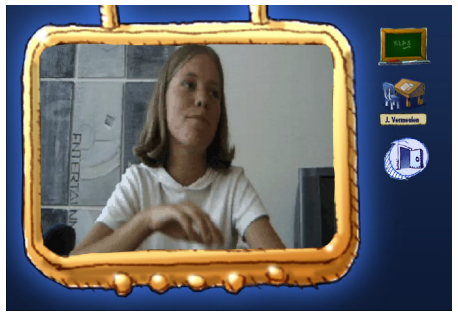

(b)

Fig. 4. a) Example view of the classroom as seen by the pupil. b) View of the pupil as seen by the teacher.

Communication between the pupil and the class happens through video-chat, similar as when using a teleconferencing system. Consequently, the pupil has to be equipped with a webcam and microphone. It is also important to provide the pupil with a means to draw attention from the teacher, for example in order to pose a question. In our system, a pupil always can ask for attention: pressing a button will flash a light and play a sound at the teacher's side. However, no audio communication will be set-up unless the teacher approves the request. This way, 
the regular teaching process won't be interrupted or distracted by background noise coming from the pupil's environment.

In order to exchange homework and corrections, a scanner and a printer have to be present as well. By clicking only one virtual button, homework and corrections can be printed, scanned or transmitted automatically. This is explained further in Section 3.2 .

Teaching in the Virtual Classroom. Analogous to the pupil the teacher can enter the virtual classroom by navigating through the virtual environment. The classroom again is represented by a fixed and static $3 \mathrm{D}$ view.

Contrary to the pupil's interface, less input is demanded from the teacher in order to not distract him/her from the regular teaching process (see Figure 4(b)). As a result, audio/video-based techniques will be employed as much as possible. Similar to the pupil's case, the same function buttons are available to quickly scan, transmit or print documents (top, right). In addition, the teacher has the possibility to capture and transmit the current content of the blackboard and record an audio/video message or even (part of) the class. These actions happen in a transparent matter by clicking one virtual button only. Section 3.2 explains this into more detail.

\section{$3.2 \quad$ Functional Aspects}

This section discusses the functional aspects of our virtual learning environment.

Diary and School Timetable. In practice, the sick child won't be able to follow all classes; often only a few hours a week.Therefore, the pupil exactly needs to know when which lessons will take place.

In our system, the teacher automatically can transmit/publish the diary or school timetable (see Figure 5(a)). Transmitted documents can be printed-out automatically and/or published in the virtual world. Figure 5(b) depicts a sick child (represented by its avatar) consulting a timetable. In addition, the sick child can send his/her schedule indicating when it would be possible to attend courses online.

Homework, Exercises and Tests. In a similar way as transmitting/publishing a diary, the sick child can do homework, exercises or take tests. First, the teacher sends the assignment as shown in Figure 5(a). Then, the sick child accomplishes the assignment. In case of exercises or tests, this can happen at the same time with the fellow pupils, increasing the feeling of being present in the class.Finally, the pupil sends the filled in assignment to the teacher.

Marking. Besides making assignments, getting feedback is also an important issue for pupils. The teacher first corrects these assignments on paper, as in case of regular pupils, after which the corrections are sent to the pupil using the system. 


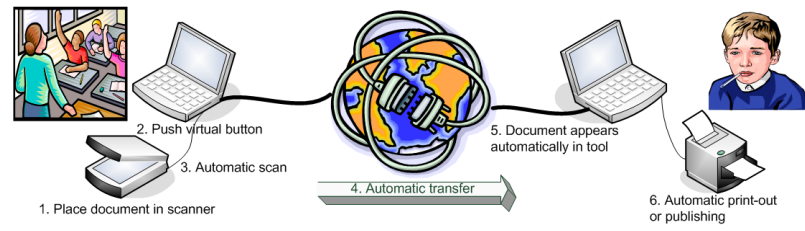

(a)

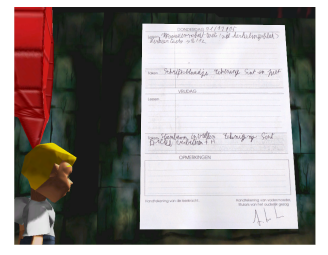

(b)

Fig. 5. a) Automatic scan, transfer, and print-out/publishing. b) 'Avatar' consulting a diary published in the virtual world.

If needful, video-chat can be employed to comment on the mistakes face-to-face. Moreover, the pupil also can participate in a class correction using live video streaming of the pupil and the class (see also next subsection). This also makes it possible for a sick child to make and correct assignments simultaneously with the fellow pupils.

Taking Lessons Synchronously. One objective of our work is to re-establish especially the communication link between the sick pupils and the regular classroom. Consequently, two issues should be considered: (i) the pupil should be able to follow the class activities simultaneously with the fellow pupils, and (ii) the pupil himself/herself should be virtually present in the classroom.

In our system, the first issue is tackled by employing a microphone and one (or more) (controllable) webcams which are mounted in the classroom. This way, the pupil can watch the general teaching (Figure 6(a)). Whenever more detail is needed, for instance important information written on the blackboard, the pupil or teacher can take a high resolution snapshot (Figure 6(b)). This happens transparently by pushing only one virtual button after which the snapshot will be visible in the virtual world.

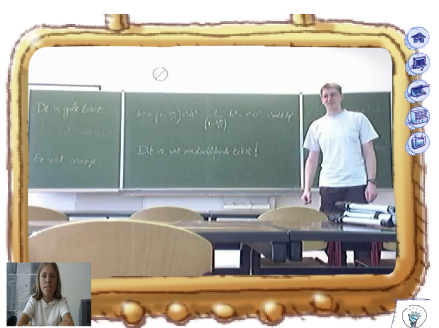

(a)

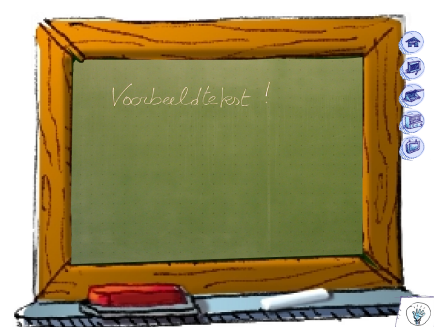

(b)

Fig. 6. a) Live view of the class as captured by the webcam present in the classroom. b) View of the blackboard. The inner part is a snapshot of the physical blackboard, taken by the digital camera or webcam present in the classroom. 
Regarding the second issue, the child's setup is also equipped with a webcam and microphone in order to be heard and seen by the fellow pupils and the teacher. By default, the child's audio is muted in order to not disturb the course with background noise coming from the pupil's environment. However, a pupil always can ask for attention - pressing a button will flash a light and play a sound at the teacher's side - but no audio communication will be set-up unless the teacher approves the request.

Taking Lessons Asynchronously. It often occurs that the sick child is unable to take a lesson using synchronous communication. Because of this we have to provide a way for exchanging information asynchronously as well. Our solution employs the metaphor of virtual books to store and retrieve information asynchronously. We distinguish between two types of virtual books: (i) public books which can be created or read by any user (e.g., to share pictures of the latest field trip), and (ii) personal books which only can be created or read by the sick child and the teacher (e.g., diary and homework) (see Figure 7).

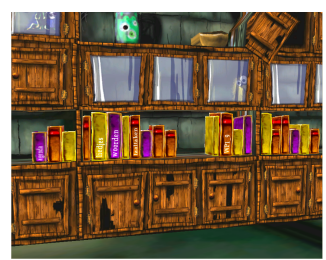

(a)

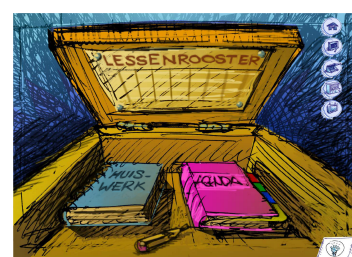

(b)

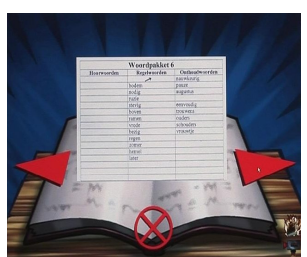

(c)

Fig. 7. Example view of the different virtual books. a) Class library used to store public virtual books. b) Pupil's personal desk containing personal books like diary and homework. c) Thumbing through a virtual book.

Social Function. Another important objective of our work is to re-establish especially the communication link between the sick pupils and the regular classroom in view of supporting social presence. The presence of a webcam, both at home/hospital and the class, makes it possible to participate in group conversations, to read out loud (together), and to practice a conversation in, for instance, French. In a similar way, fellow pupils can talk to the sick child during playtime.Furthermore, even after school pupils can meet each other in the virtual learning environment in order to communicate or share information.

\subsection{Network Architecture}

This section reports on the underlying network architecture of our system. We first describe several tasks which rely on the network. Next, we elaborate into detail which servers are responsible for which tasks. 
Figure 8 (a) portrays all tasks and different responsible servers. The protocols being used are depicted in Figure 8(b).

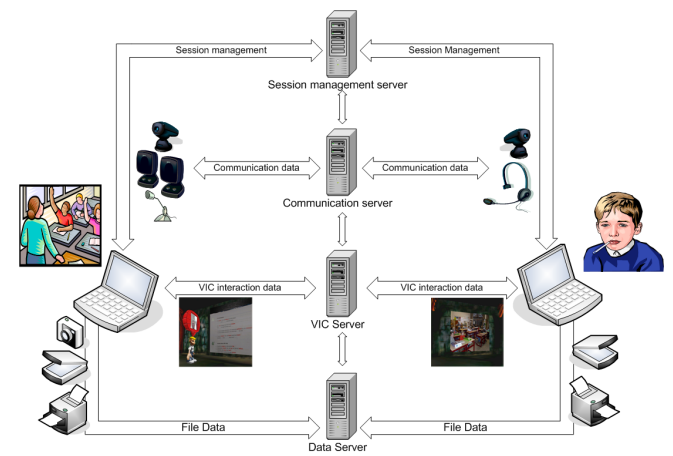

(a)

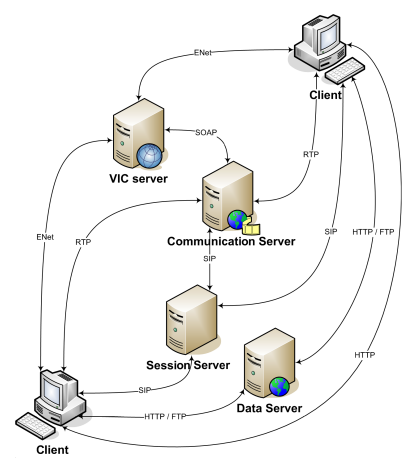

(b)

Fig. 8. Overview of the network architecture. a) All different tasks and servers. b) Employed protocols.

Tasks. In accordance with the functional aspects (see Section 3.2 four different tasks can be distinguished: (i) Session Management, (ii) VIC Interaction Management, (iii) Communication Management, and (iv) Data Transfer Management.

Upon starting the software, Session Management comes into play. It mainly is responsible for logging on/off users and taking care of authentication. After $\log$ on, the user can navigate through the virtual world by means of an avatar. At any moment, all users are being informed of each other's presence (i.e. position, mood, activity, ... ) but also of any obstacles in the world. This is managed by VIC Interaction Management. Communication Management is responsible for distributing multimedia content in synchronous mode (e.g., video-chat, attending classes). Given the real-time nature of these data streams, an obvious choice for the underlying protocol is the Real-Time Transmission Protocol or RTP [7, as it handles issues such as synchronisation and packet ordering internally. Finally, Data Transfer Management handles the transportation of data in asynchronous mode (e.g., file transfer, audio/video mails).

Servers. All tasks are being handled by a number of servers. Note that the division into different servers is done for logical reasons only; in practice all different servers can be run on only one machine.

Session Server. In first instance, the session server takes care of logging on/off and authenticating users. In addition, it is also being used to initialise A/V communication sessions and to set up file transfers. In order to manage all sessions, 
the 'Session Initiation Protocol' (SIP) 8 is being used. SIP is a text-based signaling protocol for creating, modifying, and terminating sessions with one or more participants. These sessions include internet telephone calls, multimedia distribution, and multimedia conferences. Next to this, SIP also can be used to authenticate users and it supports mobile users. This way, users can log on from different physical locations using the same account.

Communication Server. Whenever synchronous communication is needed between two parties, audio and video can be exchanged peer-to-peer. However, this is not feasible when multiple parties are involved due to the limited bandwidth. To solve this, one single stream is sent to the communication server, who at its turn will relay it to the different parties. The session server is responsible for indicating which communication server to use.

Virtual Interactive Community (VIC) Server. In short, the VIC server takes care of the entire 3D virtual environment. A first function involves transmitting the world itself. A second function concerns synchronisation between the clients. This ensures that navigation and interactions performed by clients are immediately redirected to all others. A final function relates to always storing a persistent world: adjustments to the world done by a client are propagated to all clients and stored on the server.

Data Server. The data server is used for two categories of asynchronous data. On the one hand, it stores configuration files of the users containing personal information such as A/V parameters and address lists of the community. By decentralising this kind of data, users can log on from anywhere at anytime without losing their personal settings or user interface. On the other hand, the server makes it possible to transmit and store (shared) media and files in asynchronous mode. Regarding the underlying protocols we employed Hypertext Transfer Protocol (HTTP) and File Transfer Protocol (FTP).

\section{Results}

In order to evaluate our system, field trials have been set up with the future target audience (i.e. sick children and teachers) and within authentic settings (i.e. at home, in a hospital or at school). The authentic setting required a diversity of preparatory work: methodology, instrument development (observation, interview, questionnaires, logging of data flow, ...), installation, establishing connectivity, introduction package, planning front-office support, data analysis and reporting.

Although at the time of writing not all field trials had been finished, the current tentative data were significant enough to draw conclusions regarding technical tests (i.e. hardware recommendations and network capacity). We also emphasise that evaluation of concrete user needs and functional analysis fall beyond the scope of this text as they have been conducted before and in view of our development. 


\subsection{Hardware Recommendations}

One of our initial objectives aimed at developing an affordable system. Hence, we tried to exploit commodity hardware as much as possible.

In the classroom, a digital camera is employed in order to take snapshots of the blackboard. The results clearly indicate (i) to use a resolution of 1600 x 1200 pixels (i.e. $2.1 \mathrm{Mpixel}$ ), (ii) to avoid using the flash, (iii) that colours look better on a blackboard than on a whiteboard, and (iv) to use a camera that can be controlled by software. Regarding the webcam (used for synchronous video communication), a resolution of $320 \times 240$ suffices to have a decent view and framerate (25fps). Tests also pointed out that it is even possible to capture a film that is shown on the classroom television. Concerning audio, the pupil easily can make use of a headset or the microphone integrated into the webcam. The teacher is advised against using a headset, but to use a wireless microphone together with fixed speakers. This requires the need for acoustic echo cancellation (AEC), either built in in the microphone or our software implementation 9]. Finally, the personal computer itself, needs to have a 3D accelerator graphics card in order to fluently visualise the $3 \mathrm{D}$ environment.

\subsection{Network Capacity}

Regarding bandwidth, the real bottleneck is streaming live audio and video. To keep network traffic to a minimum this should be compressed before streaming. Our tests pointed out that when using the H.263+ codec [10 for compressing video (comprised of $320 \times 240$ pixels at $25 \mathrm{~Hz}$ ), $128 \mathrm{~kb}$ bandwidth is needed. This is quite acceptable as most people and schools (in the Flemish setting) own a xDSL or cable connection (download speed: $4.4 \mathrm{Mb}$, upload: $192-256 \mathrm{~kb}$ ).

\section{Conclusions}

Existing ICT-based solutions to foster communication for long term ill children primary focus on the re-entry of the child in the community and school rather than pre-empting this process by bringing the community and school to him, and have weaknesses including absence of social involvement, high cost of developing, and mobility issues.

We presented a system that is based on concrete user needs, is educational sound and relevant, and offers a scalable and affordable solution. It comprises a technical solution that helps to establish high quality involvement of the long term sick children in a communication based scenario between the place where the child stays/has been moved and the original classroom/school setting. The communication provisions build on audio/video links (3D, very high to very low quality video links, synchronous versus asynchronous solutions), and help to support educational scenarios (e.g. a one-to-one scenario) that support learning processes. Furthermore, we balanced the choice for high end solutions with the need to develop a scalable and affordable solution. 
We believe our system (re)establishes high quality communication between the sick children and their regular school and classroom learning environment, even after school.

Future Work. In the near future, a second iteration of the field trials is planned taking into account all mentioned recommendations. This field test will also involve sick children, and on the other hand teachers, school friends, and peers. Next to an educational evaluation, also the user friendliness of the user interface will be evaluated.

\section{Acknowledgements}

This study was conducted in view of the ASCIT-project (Again at my School by fostering Communication through Interactive Technologies for long term sick children) financed by the Flemish Interdisciplinary institute for Broadband Technology (IBBT). We gratefully express our gratitude to the European Fund for Regional Development (ERDF) and the Flemish Government which are kindly funding part of the research at the Expertise Centre for Digital Media. Many thanks go also to Xemi Morales for his artistic contribution.

\section{References}

1. The Learning Collaborative, The National Center for Electronically Mediated Learning: The PEBBLES project. (http://www.pebblesproject.org)

2. Starlight Starbright children's foundation: Starbright World. (http://www . starbrightworld.org)

3. Battles, H., Wiener, L.: Starbright World: Effects of an electronic network on the social environment of children with life-threatening illnesses. Children's Health Care (2002) 47-68

4. Jonge Kamera v.z.w.: Jonge kamera. (http://www.jongekamera.be)

5. Lombaert, E., Valcke, M.: Education for long-term sick children: Towards an integrated it-solution. To be published in the Computer Assisted Learning conference (CAL '07) (2007)

6. Quax, P., Flerackers, C., Jehaes, T., Lamotte, W.: Scalable transmission of avatar video streams in virtual environments. In: Proceedings of the 2004 IEEE International Conference on Multimedia and Expo (ICME 2004). (2004) 631-634

7. Schulzrinne, H., Casner, S., Frederick, R., Jacobson, V.: RTP: A transport protocol for real-time applications, RFC 3550. Technical report (2003)

8. Rosenberg, J., Schulzrinne, H., Camarillo, G., Johnston, A., Peterson, J., Sparks, R., Handley, M., Schooler, E.: SIP: Session Initiation Protocol, RFC 3261. Technical report (2002)

9. Schobben, D.E.: Real-Time Adaptive Concepts in Acoustics: Blind Signal Separation and Multichannel Echo Cancellation. (Springer)

10. Bormann, C., Cline, L., Deisher, G., Gardos, T., Maciocco, C., Dewell, D., Ott, J., Sullivan, G., Wenger, S., Zhu, C.: RTP Payload Format for the 1998 Version of ITU-T Rec. H.263 Video (H.263+), RFC 2429. Technical report (1998) 